\title{
Los ritmos del Pachakuti. Cómo conocemos las luchas de emancipación y su relación con la política de la autonomía*
}

\author{
Raquel Gutiérrez Aguilar
}

El trabajo analiza la cuestión de la emancipación social en Bolivia, sus desafíos, trayectorias y dificultades. Recupera algunas experiencias de las acciones que los hombres y mujeres de base emprendieron durante el primer periodo de gobierno de Evo Morales (2006-2009) y reflexiona acerca de sus posibilidades políticas.

PALABRAS CLAVE: emancipación, formas de la política, formas de lo político, Bolivia, Pachakuti, transformación social

\section{The Rhythms of Pachakuti. How we Get to Know Emancipatory Struggles and Their Relation-} ship with the Politics of Autonomy

This work analyses the issue of social emancipation in Bolivia, its challenges, trajectories and difficulties. It recovers some experiences of the actions taken by men and women of the base during the Evo Morales government of 2006-2009 and reflects upon their political possibilities.

KEYWORDS: emancipation, forms of the political, forms of politics, Bolivia, Pachakuti, social transformation

RaQuel GutiérRez Aguilar: Centro de Estudios Andinos y Mesoamericanos, A. C., Distrito Federal, México raquel.gutierrezaguilar@gmail.com 
$\mathrm{E}$ n este trabajo profundizo en algunos de los argumentos que desarrollé en Los ritmos del Pachakuti (2009). En particular, me pregunto sobre las posibilidades y las formas de las luchas de emancipación desde la realidad en Bolivia, aunque no de manera exclusiva. La emancipación social, sus cursos concretos, sus desafíos y dificultades continúan en los debates contemporáneos, sobre todo en relación con la tensión contradictoria entre los eventuales gobiernos progresistas - sus acciones y perspectivas - y la gigantesca capacidad social, autónoma y directa, para intervenir en asuntos públicos que incumben a todos. Esto fue visible entre 2000 y 2005, los años de los levantamientos y movilizaciones indígenas y populares más potentes que hicieron colapsar una parte importante del orden económico y político colonial neoliberal en ese país. Parto de un punto complicado: la relación entre la emancipación social y la cuestión del poder en tiempos de gobiernos progresistas. La tensión entre ambas quedó planteada en una reunión de la Coordinadora del Agua y de la Vida en Cochabamba, realizada en marzo de 2006 - a dos meses de que Evo Morales asumiera la presidencia de Bolivia con una abrumadora mayoría de votos-, en la que el problema se expresó de la siguiente manera: "La cuestión de cómo ejercer el gobierno es actualmente el problema del Movimiento al Socialismo (MAS); la cuestión que sigue estando frente a nosotros es el problema del poder, de su disolución y trastocamiento".

En primer lugar, tal afirmación mantiene a la vista la distinción - absolutamente práctica y cuyo acercamiento muchas veces es más bien teórico- entre ocupar cargos públicos —incluso la presidencia de un país, con las consecuentes prerrogativas para decidir sobre asuntos públicos específicos y la obligatoriedad de ceñirse a normativas heredadas, a formas rígidas institucionales y al cumplimiento de formatos administrativos viejos-y la mucho más significativa y compleja dificultad de establecer los límites a las viejas prerrogativas de mando y monopolio sobre la decisión pública desde la sociedad llana, con base en la deliberación y el acuerdo común. Este asunto se entiende como una trayectoria colectiva, como un itinerario a hilvanar, y no como una finalidad $\mathrm{u}$ objetivo que pueda alcanzarse por completo en algún momento de la historia. Así, abre desde "la población sencilla y trabajadora" ${ }^{1}$ un vasto campo de problemas políticos a solucionar y discute la noción misma de lo político. En segundo lugar y en concordancia con lo anterior, la formulación sitúa en su justo lugar el problema del sujeto de la emancipación social cuando distingue entre los conglomerados y cuerpos asociativos de la sociedad trabajadora -que tienen ante sí el problema de la disolución y escape del poder-imposición -22 y la peculiar corporación que ocupa temporalmente el aparato de gobierno. Además, de manera implícita, al establecer ante sí nuevas tareas en relación con la disolución del poder-imposición se coloca en primer plano el problema del cuidado y expansión de las capacidades colectivas propias que permiten des-sujetarse de las tramas de dominación, deshilvanándolas.

En los momentos eufóricos de inicios de 2006, cuando comenzaba el régimen de Evo Morales y

\footnotetext{
1 "Nosotros, la gente sencilla y trabajadora" fue y es la manera en que la Coordinadora de Defensa del Agua y de la Vida - una articulación política flexible, estable y no institucional que distintas organizaciones sindicales y sociales y grupos de activistas y profesionales produjeron durante la "guerra del agua" en Cochabamba- se describe y enuncia a su interlocutor. De esta forma, establece en cada ocasión su lugar de enunciación: en primera persona, por fuera del Estado y desde el trabajo.

${ }^{2}$ Vale la pena tener en cuenta de manera permanente el contraste entre dos significados del término "poder": como prerrogativa de ejercer mando sobre los asuntos sociales o como fuerza social - detentada por particulares - para instituir destinos y organizar formas específicas de producción y usufructo de la riqueza social, formulación que contrasta drásticamente con otra acepción del término que lo relaciona con la capacidad —individual y colectiva - de producir, generar y regenerar la vida en sus múltiples dimensiones construyendo y afianzando modalidades autónomas de autorregulación. Estos sentidos contradictorios han sido abordados por diversos actores, en particular por John Holloway en Cambiar el mundo sin tomar el poder (2001). Sobre el tema y de manera similar, aunque independiente, hay algunos argumentos en mi trabajo: $; A$ desordenar! Por una historia abierta de la lucha social (Gutiérrez, 2006).
} 


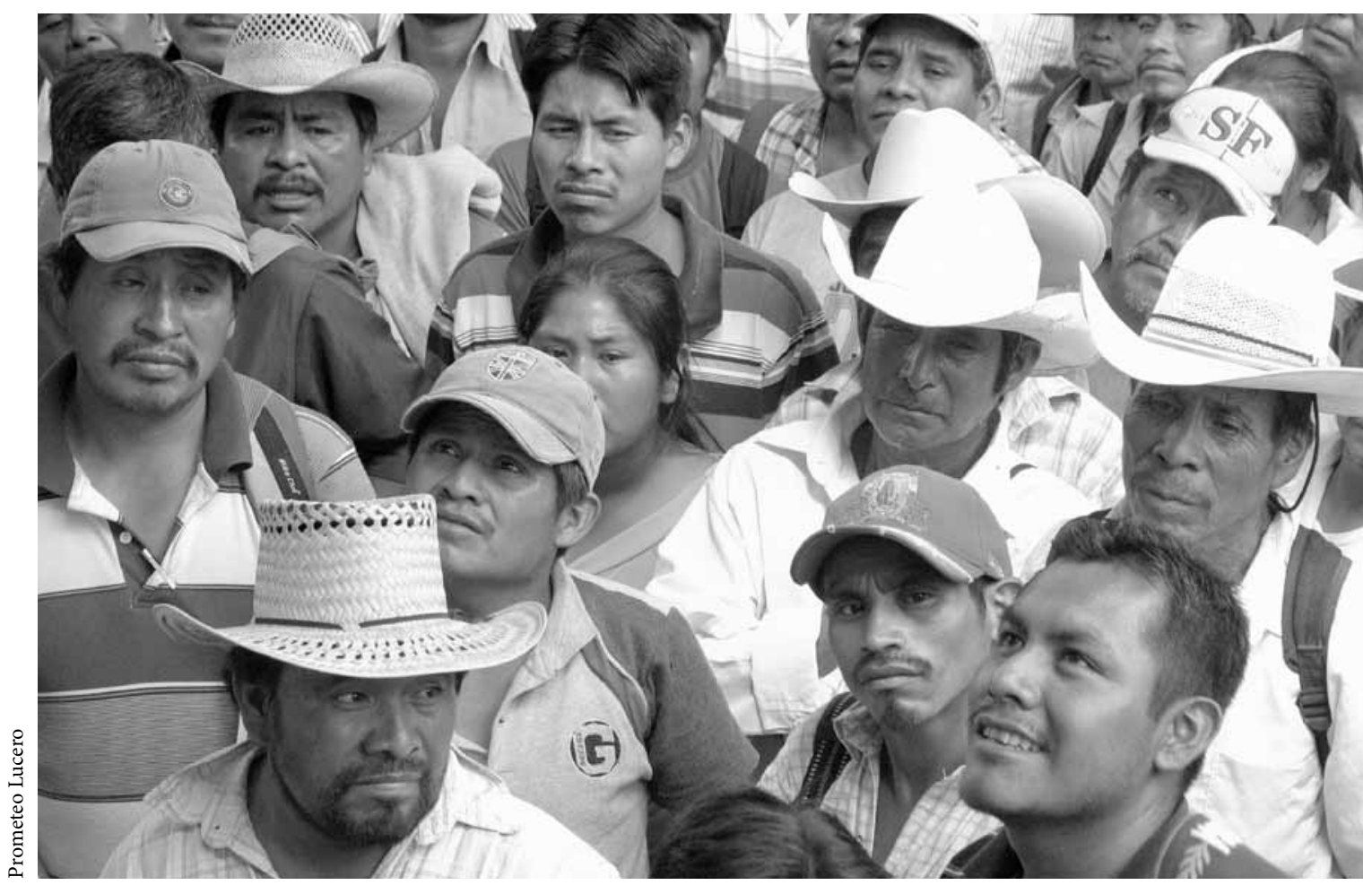

Indígenas de diversas comunidades y municipios se concentran en Tlapa de Comonfort, Guerrero, para protestar contra las altas tarifas de energía eléctrica, 2008

habían pasado apenas seis meses desde la última gran oleada de levantamiento y rebelión, ${ }^{3}$ escuché a compañeras aymaras del sindicato de vendedoras de pescado de la ciudad de El Alto expresar lo siguiente:

Evo es como el marido que se casa con todos nosotros, con Bolivia, el día de las elecciones. Él tiene su tarea, nosotros tenemos la nuestra. Que no se meta con nosotras, que no venga a decirnos qué hacer. Nosotras ya hemos aprendido qué tenemos que hacer. Él tiene que estar ahí ocupándose de que los extranjeros y los q’aras no molesten. Nosotras vamos a hacer todo lo demás.

\footnotetext{
${ }^{3}$ La última movilización de la ola de levantamientos contra los gobiernos neoliberales ocurrió en mayo-junio de 2005. "La población boliviana sencilla y trabajadora" fue capaz, en el contexto de la renuncia del presidente sustituto de entonces, Carlos Meza, de desarmar una maniobra política de los partidos tradicionales de derecha que pretendían establecer su propio proyecto sucesorio y mantener el control del gobierno.
}

Las compañeras también insistían en la cuestión decisiva de que eran ellas mismas quienes debían ocuparse de transformar la vida cotidiana y las relaciones sociales en general. Lo más elocuente es la afirmación que representaban con la metáfora familiar del casamiento: "que [Evo, pensado como "marido" de la sociedad boliviana] no venga a decirnos qué hacer; él tiene su tarea, nosotras la nuestra”. Ante mi pregunta insistente de qué era lo que nosotras tenemos que hacer, las compañeras hablaban de manera difusa de diversos anhelos: "más escuelas y opciones para nuestros hijos, los jóvenes", "mejorar" la vida cotidiana, etc. Consideraban que para afrontar tales asuntos, el gobierno debía "apoyar", pero enfatizaban un aspecto fundamental: "a nosotras nos toca cuidar que Evo no se desvíe. Él tiene que apoyarnos a nosotras, en lo que vayamos decidiendo entre todos". Esta sencilla manera de expresar un 
asunto político mayor sobre quién es a fin de cuentas el titular de la soberanía social, quién detenta la prerrogativa de decidir sobre los asuntos que a todos o a muchos incumben, me parece muy fértil. Refleja la manera de entender desde una perspectiva femenina popular-comunitaria la relación entre quien será ocasionalmente "el encargado del gobierno" y las partes que conforman el "cuerpo social": "él tiene que estar ahí para apoyar lo que nosotros vayamos decidiendo".

Desde la perspectiva que sostengo, estas dos formulaciones insinúan una manera concreta y particular, práctica - en contraste con las formas abstractas y generales, teóricas, de los "planes de gobierno" o los "programas" - de proyectar un horizonte de sentido autónomo que ilumina los propios pasos que pueden ser dados en común. Más adelante presentaré algunas consideraciones acerca de las luchas de emancipación elaboradas desde estas búsquedas. Lo que las compañeras de El Alto diseñaban como perspectiva abierta de transformación política no fue lo que ocurrió en los años siguientes, al menos no de manera fluida. Para comprenderlo, veamos en un primer momento y de manera muy breve qué hizo el gobierno del MAs durante su primera gestión. Después mencionaremos algunas de las iniciativas que se imaginaron y se intentaron desde la sociedad en relación con la "reapropiación" de la riqueza social, natural y pública, y finalmente reflexionaremos sobre tales experiencias para bosquejar ideas más generales.

El primer periodo de gobierno de Morales (20062010) estuvo marcado por cuatro líneas de actividad política: i) la convocatoria y realización de una Asamblea Constituyente que, pese a conservar un formato liberal para la selección de los diputados constituyentes, permitió un amplio debate nacional sobre algunas de las modificaciones formales más importantes del Estado desde la perspectiva indígena y popular; ${ }^{4}$ ii) la renegociación de los contratos de explotación de los hidrocarburos con las compañías transnacionales más poderosas que hasta entonces se habían erigido en casi únicas dueñas de las riquezas del subsuelo boliviano; ${ }^{5}$ iii) la neutralización parcial de la amenaza política de las elites regionales terratenientes agroexportadoras del Oriente, que desde 2005 introdujeron al debate público el tema de la "autonomía regional" como pretexto para anular cualquier transformación económica-política, al menos en su región, y conservar sus privilegios, ${ }^{6} \mathrm{y}$ iv) la cooptación y subordinación tendencial de las voces y ensayos asociativos que nacieron o se consolidaron y se expandieron durante los años de las rebeliones a lo largo y ancho del país. ${ }^{7}$

Estas acciones y medidas políticas se entrelazaron con la puesta en marcha de algunas "políticas públicas de transferencia de recursos" según lo sugiere y alienta el Banco Mundial. En particular, se instituyó

\footnotetext{
${ }^{4}$ Lamentablemente, la Constitución Política que resultó de esa Asamblea mantuvo casi intacta una parte sustancial de los fundamentos materiales del Estado liberal: la gran propiedad de la tierra en Oriente y el respeto a la representación política delegada y mediada por la ley y las instituciones estatales. Véase Tapia, Mokrani y Chávez (2007). Puede hallarse una discusión más detallada de las primeras medidas políticas del gobierno del MAs en Gutiérrez y Mokrani (2009).

${ }^{5}$ Morales y su gobierno establecieron nuevos términos para la relación entre el Estado y las transnacionales bastante más favorables para el primero, con el pomposo nombre de "nacionalización de los hidrocarburos". Véase Gutiérrez y Mokrani (2009).

${ }^{6}$ El gobierno consiguió dicha neutralización parcial a través de una confusa mezcla de medidas políticas y administrativas de cooptación, concesión y disminución de presupuestos públicos que han sido duramente criticadas, aunque admitidas pese a la inconformidad, desde los movimientos sociales de las regiones orientales. El desarrollo de los momentos más tensos en este conflicto ocurrió durante 2008. Elaboré algunas crónicas analíticas de los sucesos que derivaron en la Masacre de Pando para el centro de información Americas Program del Center for International Policy. Pueden consultarse en <www.cipamericas.org $>$.

${ }^{7}$ Dicha línea de cooptación y control está en la base de la formación de la llamada Coordinadora Nacional para el Cambio (Conacam), instancia organizativa tutelada por el gobierno que aglutina a los "movimientos sociales" para garantizar su adhesión y apoyo a las decisiones masistas.
} 
el "Bono Juancito Pinto" para los estudiantes de menores recursos y programas de apoyo a mujeres "emprendedoras". En contraste con estas acciones que no ofrecen demasiadas novedades políticas, miremos un poco hacia dos iniciativas, lamentablemente truncas, que desde algunos espacios y cuerpos de la sociedad llana se insinuaron como posibilidad.

\section{II}

Primero fue el intento de "desprivatización" de la entonces aerolínea "de bandera" Lloyd Aéreo Boliviano, que los trabajadores de la empresa imaginaban como paso inicial para abrir la posibilidad de autogestionar las operaciones de la misma. "El Lloyd", como se le conocía en Bolivia, durante muchos años tuvo su sede en Cochabamba. Los pilotos, las azafatas y demás personal de la empresa conocieron $-\mathrm{y}$ muchos participaron en- la ola de luchas y levantamientos en torno a la defensa del agua que comenzó en el 2000. En particular, presenciaron y participaron en deliberaciones sobre un tema decisivo: la reapropiación social de la riqueza común. La discusión central fue acerca de la gestión y el usufructo legítimo del agua. En aquellos años, el tema se discutía en Bolivia de maneras diversas y argumentos de todo tipo se diseminaron tras la llamada "guerra del gas".

Enmedio de aquel ambiente de movilización y discusión, más aún, de disposición colectiva a no ceñirse a los límites que las leyes e instituciones heredadas establecían como admisibles, y de deliberación colectiva acerca de otras posibilidades, Evo Morales asumió el cargo de presidente del país en enero de 2006. En tales circunstancias, los trabajadores del Lloyd elaboraron un plan de "reapropiación" y "tendencial autogestión" de su empresa, que

\footnotetext{
8 Véanse los capítulos I y III de Gutiérrez (2009) para una discusión más amplia sobre los caminos y modos para la "reapropiación social de los bienes comunes".
}

consistía en: i) trazar un camino de "desprivatización formal" por medio del juicio al "socio capitalista” por el incumplimiento de la mayor parte de las cláusulas del contrato inicial de "riesgo compartido". 9 Por caminos legales proponían disolver la desfavorable "sociedad" que se había conformado en 1995 durante el primer gobierno de Sánchez de Lozada; ii) que los trabajadores se "desafiliaran" de manera colectiva del sistema privatizado de pensiones - llamadas afores en México y AFP en Bolivia-, dado que la primera acción iba a "secar" las fuentes de financiamiento para capital de operación y con esos recursos se crearía un fondo común para echar a andar la empresa por cuenta propia. El conjunto de cuestiones específicas no estaba bien definido: si mediante esa "aportación" se convertían en nuevos socios del Estado - por tanto, en "dueños" parciales de la empresa-, si ésta volvía a adquirir un carácter público y el Estado buscaría posteriormente otros fondos para asegurarles su jubilación, etc. Lo que sí estaba claro, y llenaba de entusiasmo a todos, es que

${ }^{9}$ La cuestión de la "desprivatización" se abordaba de la siguiente forma: las antiguas empresas públicas habían pasado a manos de empresarios transnacionales, en particular Lloyd, mediante "contratos de riesgo compartido": los "socios corporativos" invertían sus capitales y obtenían el control casi total de la gestión de la empresa a cambio de prácticamente nada, en tanto "los ciudadanos bolivianos mayores de 18 años hasta 1995" mantenían la titularidad individualizada de unas acciones de las empresas hipotéticas y fantasmagóricas que a su vez se concentraban en los fondos de pensiones de reciente creación. Pura perversión financiera, pletórica de términos tecnocráticos y de cálculos matemáticos tan complejos como insignificantes y tramposos. Los trabajadores del Lloyd proponían recorrer el camino a la inversa: aprovechar las inconsistencias y debilidades, como los incumplimientos corporativos a los contratos, para deshacer paulatinamente los formalismos de la privatización. Se pensaba así pues desde la expulsión de la empresa Bechtel en 2000 -titular de la concesión del agua en Cochabamba- el Estado boliviano había sido demandado ante los tribunales internacionales ad hoc - el Tratado Interamericano de Asistencia Recíproca-, donde los pillos de cuello blanco demandan pagos millonarios por "ganancias no devengadas" ( $\mathrm{j}$ sic!) que pagan las poblaciones trabajadoras. Estos asuntos traspasaron las paredes de los gabinetes de los expertos y eran moneda corriente en la discusión pública en Bolivia. Era claramente parte sustancial de aquellos tiempos extraordinarios. 
imaginaban que "se podía hacer otra cosa" y había una gran disposición a transitar ese camino.

En marzo de 2006, tras acordar las líneas generales del "rescate" y tendencial "reapropiación" del Lloyd, los trabajadores decidieron proponer sus ideas a Morales y su gobierno para que diseñaran un plan de acción común. Iban a hablar con él en tanto lo consideraban un aliado; además, se requerían apoyos desde el propio gobierno, sobre todo respaldo político. Estaban eufóricos. Los más críticos a la economía liberal explicaban a los demás el conjunto de virtudes de su plan: se recuperaba una empresa antes privatizada y "de pasada" se quitaba de las manos del capital financiero al menos una parte del ahorro de los trabajadores que había sido usufructuado privadamente por esas otras corporaciones. Ellos estaban dispuestos a trabajar mucho, confiaban en que podían abrir, con cautela y paso a paso, un camino nuevo para reapropiarse de la empresa y ensayar formas de autogestión. ${ }^{10}$

Nada de lo anterior ocurrió. Evo Morales los recibió, los escuchó, les dijo que iba a preguntar a sus asesores internos y externos sobre las posibilidades de llevar a cabo lo que exponían y no volvió a recibirlos. El gobierno de Morales no quiso pensar, para nada, en dicha posibilidad. Los trabajadores del Lloyd, apoyados por otros sectores trabajadores y populares de la ciudad de Cochabamba y por la Coordinadora del Agua y de la Vida, se movilizaron varias veces durante marzo exigiendo una discusión de fondo sobre su propuesta. Querían un diálogo público entre Evo y su gobierno y los trabajadores. Su exigencia nunca fue atendida. Casi a fin de mes, los trabajadores, con apoyo de vecinos y activistas de Cochabamba, tomaron el aeropuerto y fueron desalojados de manera violenta. Se les amenazó con la cárcel y el gobierno armó una campaña de propaganda que insistía en que "el plan de los pilotos no era factible". Así, a dos meses de ocupar la silla presidencial, el

${ }^{10}$ Vale la pena notar que en este otro contexto, laboral, especializado y urbano, se aplicaba también la idea del discurso de las mujeres aymaras: "ellos, los del gobierno, que ayuden a que las transnacionales no molesten; nosotros hacemos todo lo demás”. gobierno estableció a quien consideraba que pertenecía la prerrogativa de tomar las decisiones. El Lloyd dejó de volar meses después.

Dejamos aquí el ejemplo, pues no se trata de analizar que la ocupación de cargos públicos y la disposición de cierto poder de imposición ciega a los excompañeros. Lo que se busca es recoger algunas ideas de la sociedad llana — que bullían en Bolivia en aquellos momentos extraordinarios-, documentar los límites de esta especie de "reformismo desde abajo" - si no está claramente planteada la necesaria inversión de la titularidad de la decisión política, las precisiones necesarias para dar vida al "mandar obedeciendo" - y analizar lo que podemos nombrar como una política de y desde la autonomía y las dificultades que existen para ella.

Si miramos con cuidado, notamos que los trabajadores del Lloyd sugerían una manera particular para avanzar poco a poco, profundizando el camino de la política autónoma contra el capital y el Estado que había brotado con fuerza en Cochabamba en relación con el agua unos años atrás. No tenían una solución, pero bosquejaban un camino y, en aquellos momentos, confiaban - equivocadamente- en que el gobierno de Morales podía ser un aliado, un apoyo mínimo. Nunca pensaron que sería un obstáculo para sus proyectos. En las condiciones de Bolivia en 2006, no supieron cómo ni tuvieron fuerza suficiente para superar ese límite. ${ }^{11}$

Una segunda experiencia, en la que se hizo evidente la versátil política de la autonomía, surgió durante algunos años en el altiplano aymara a través de los llamados “Ponchos Rojos”. No es fácil contestar "qué

\footnotetext{
${ }^{11}$ Hacía menos de un año que las principales acciones de lucha y resistencia se coordinaban abierta y directamente también con Morales. Era, antes que un presidente, un amigo con quien los trabajadores de Cochabamba creían que podían llegar a un acuerdo. La actitud tanto de los trabajadores de Lloyd como de la propia Coordinadora del Agua era llamar al diálogo público para mostrar la viabilidad de sus propuestas y para mantener abierto el sentido común y la disposición colectiva hacia la transformación de las relaciones sociales. Confiaban en que podrían avanzar por el camino de la deliberación pública y la elaboración de objetivos autónomos que había tenido tan buenos resultados en años anteriores. Poco a poco chocaron con la realidad: el gobierno masista
} 
son" los Ponchos Rojos. Seguramente la respuesta que delinearé no puede ser completa. De inicio, me guío por quienes se presentan, intermitentemente, con ese apelativo. En un primer acercamiento, los Ponchos Rojos son en su mayoría comunarios - varones- aymaras, tanto autoridades como "bases", que durante los años de levantamientos y prolongados bloqueos se presentaban a las asambleas y cabildos vistiendo el poncho rojo con negro que es la indumentaria masculina tradicional de tiempos de guerra. Que todos vistan el poncho rojo alude a un fuerte simbolismo comunitario que va estableciendo el significado de los tiempos, no sólo mediante palabras, sino recurriendo a un rico acervo lingüístico y comunicativo. Así, el hecho de que las autoridades comunitarias se presentaran juntas vistiendo sus ponchos rayados y portando sus símbolos de autoridad -el chicote, el pututu - se convirtió en una forma de imponer una presencia amenazadora que todos entendían: amigos y enemigos. Cuando Evo Morales llegó al Palacio Quemado, muchos aymaras no masistas, conocidos y respetados veteranos de los levantamientos, comenzaron a presentarse ante Morales o ante sus representantes vistiendo justamente sus ponchos rojos, con lo que expresaban que ellos deciden por sí mismos y que tienen fuerza para llevar adelante sus decisiones. ${ }^{12}$

En este primer nivel, Ponchos Rojos es el nombre de la fuerza autónoma colectiva que se presenta públicamente para hacer patente su voluntad y disposición de lucha porque sus integrantes comparten

estaba mucho más interesado en su propia consolidación como gobierno que en la actividad persistente de proyectar, discutir y ejecutar rutas para reapropiarse en común de lo privatizado. En este sentido, la metáfora de las mujeres aymaras es muy fértil: ocurre algo similar a cuando, después del matrimonio, el "marido", que hasta entonces había sido un cortés enamorado, se vuelve "otra persona" y busca controlar las acciones, pensamientos y tiempos de "la esposa". Ésta suele caer en un profundo desconcierto, pues efectivamente quería casarse, pero de ninguna forma quería - ni buscaba - lo que obtiene.

12 Por esta razón los Ponchos Rojos son considerados, sobre todo en las crónicas periodísticas, como una "milicia aymara" cercana a Evo Morales, lo que era definitivamente falso durante los primeros años de gobierno. un modo de vivir y de pelear que se produce y se entiende, también, con lenguajes no verbales. En un segundo nivel, los Ponchos Rojos más notables y reconocidos eran algunos de los comunarios más politizados y combativos de la región de Omasuyos, relacionados y representados hasta cierto punto - que no organizados de manera rígida- por Eugenio Rojas, luchador aymara y alcalde de Achacachi durante los años en que no hubo policía ni cárcel en tales territorios. En esta clave, los Ponchos Rojos son una más de las "anomalías" asociativas que se gestaron y se hicieron visibles durante los años rebeldes. No "cabe" una explicación de lo que eran bajo cánones más tradicionales — donde se determinan criterios de pertenencia-, son más bien la institución de un sentido de inclusión: somos Ponchos Rojos quienes somos y nos presentamos así, comunarios aymaras en apronte y con disposición de pelea — definición ambigua y casi tautológica si el afán es identificar-.

Los Ponchos Rojos reconocieron la autoridad de Evo Morales durante los primeros años de su gestión. Le expresaron su disposición a apoyarlo en la confrontación contra la oligarquía del Oriente y, al mismo tiempo, le manifestaron su desconfianza y molestia en relación con muchas de las prácticas políticas y de las decisiones que se tomaron desde el gobierno. Sobre la disposición a apoyar a Morales en la disputa contra la oligarquía, todavía se recuerda la llegada de los Ponchos Rojos a Santa Cruz en ocasión de las Fiestas Patrias, en agosto de 2006, aparición que literalmente acalambró a la elite regional. En aquella oportunidad, Morales pidió explícitamente a los Ponchos Rojos que participaran en la celebración y ellos accedieron. En el juego simbólico boliviano, los Ponchos Rojos desfilaron por Santa Cruz exhibiendo tanto wiphalas - banderas indígenas - como insignias tricolores - la bandera boliviana oficial—, algo que jamás habrían hecho en La Paz. Fue un interesante modo de decir en público a los gamonales del Oriente que ellos también estarían presentes en las luchas de los demás bolivianos contra la entonces incipiente, si bien ya violenta, 
estrategia terrateniente de conservar privilegios y propiedades promoviendo la escisión del país a título de "autonomía regional" o "departamental".

Respecto de la tensa desconfianza, manifiesta durante mucho tiempo, de los Ponchos Rojos hacia el gobierno, pueden rastrearse algunas declaraciones públicas de sus principales voceros y, sobre todo, formas variadas de explicitar la voluntad práctica de mantenerse al margen del partido oficial y del gobierno: en la organización de los aniversarios de las fechas más notables de los levantamientos, en la postura asumida en ocasión de los múltiples comicios que se llevaron a cabo durante la primera gestión de Morales, ${ }^{13}$ en el trabajo político y simbólico que echaron a andar bajo la idea de la necesidad de "reconstrucción de los cuerpos de Tupak Katari y Bartolina Sisa" (Mamani, Choque y Delgado, 2010), etc. El gobierno nunca estuvo cómodo con este espacio de enunciación propia y autónoma que el movimiento político indígena había construido para sí mismo. $\mathrm{Pu}$ so sus mayores esfuerzos en anularlo por la vía de la cooptación y en 2009 obtuvo una victoria sobre la política autónoma de estos comunarios aymaras: convirtió a Eugenio Rojas en senador por el MAs.

Encontramos en este ejemplo otra forma de bosquejar una perspectiva política autónoma, más pausada, centrada en el cuidado y consolidación de la fuerza propia, distinta aunque emparentada con las propuestas de los trabajadores de Cochabamba. Entre 2006 y 2009, los Ponchos Rojos se presentaron en el espacio y debate públicos intermitentemente cuidando siempre su autonomía política. Intervinieron con su presencia amenazadora en el mapa de fuerzas confrontadas que se trazó en Bolivia hasta 2008 en los momentos más difíciles, cuando la oligarquía terrateniente, las corporaciones transnacionales y el gobierno estadounidense hicieron los

\footnotetext{
${ }^{13}$ Durante los primeros tres años de gobierno del mas hubo al menos cuatro procesos electorales y de auscultación a la sociedad por la vía del voto: la elección de los diputados constituyentes en 2006, el referéndum revocatorio en 2008, que se enlazó con la posterior elección directa de autoridades departamentales, y la consulta sobre la aprobación de la Nueva Constitución Política del Estado.
}

mayores esfuerzos por limitar las aspiraciones sociales de la población trabajadora del Oriente y por desestabilizar y disminuir la capacidad de gobierno del mas. De manera simultánea, desarrollaron su agenda de deliberación y producción de horizonte político, más allá del gobierno y usando sus formas y lenguajes propios: tal es, en cierta medida, el significado de la llamada tarea de reconstitución del cuerpo político de Tupak Katari y Bartolina Sisa que han echado a andar. Han criticado duramente al gobierno del MAs, apoyando las luchas que se han producido desde abajo para impedir la ejecución de decisiones gubernamentales equivocadas y contrarias a los deseos y decisiones de las comunidades y pueblos. Durante algunos años se mantuvieron a sí mismos como presencia política autónoma en la retaguardia, y quizás, pese al debilitamiento que supone para esta postura que una de sus figuras más visibles haya decidido ocupar un cargo público con el MAs, el tejido comunitario aymara, más allá de los Ponchos Rojos, será capaz de producir novedades políticas en un futuro próximo.

\section{III}

A partir de los elementos presentados, desarrollaré una reflexión más general sobre algunos asuntos de la política autónoma y de las luchas de emancipación. Presentaré brevemente algunas consideraciones abstractas, para discutir por último los temas que considero más complicados.

En tanto la emancipación social es, ante todo, una cuestión práctica, un asunto que hace al mosaico móvil de actividades que se despliegan mayormente en momentos de enérgico despliegue de la confrontación social, antes que una "teoría" —entendida como conjunto fijo y exterior de argumentos ordenados y sistemáticos-, requerimos de una estrategia teórica para entender los rasgos de emancipación de los movimientos y rebeliones sociales recientes en sus alcances históricos. A diferencia del significado clásico 
de la palabra teoría, una estrategia teórica no tiene la pretensión de encubrir, en nombre de cierta noción de objetividad, al sujeto que teoriza. Más bien, busca presentar los sucesos como producción práctica y reflexiva de personas situadas socialmente, que asumen intencionalidades políticas determinadas, ya sean señaladas de manera explícita o implícitamente asumidas. La estrategia teórica que propongo no se inserta en la tradición que privilegia la producción de conocimiento objetivo, sino en la que auspicia la comprensión práctica ${ }^{14} \mathrm{del}$ acontecimiento social de quiebre, resistencia e impugnación del orden social por aquellos quienes lo producen. Asumo, por lo mismo, dos órdenes o niveles lógicos para la comprensión de la emancipación: el primero, y fundamental, tiene que ver con las propias prácticas de emancipación inscritas en la actividad política concreta de los distintos conglomerados de hombres y mujeres que, con sus acciones de levantamiento y movilización en Bolivia, abrieron nuevas perspectivas para producir y pensar

\footnotetext{
14 Trabajé más sistemáticamente la distinción entre conocimiento objetivo y comprensión práctica en mi tesis de maestría en filosofía de la lógica, aunque con un elevado grado de abstracción que impide que el contenido sea fácilmente puesto a consideración de especialistas de otras disciplinas. En aquella oportunidad estudié la relación entre la naturaleza de las proposiciones de la aritmética y la noción de número en dos filósofos (Mill y Frege) y dos matemáticos (Cantor y Dedekind). Encontré una distinción muy clara entre lo que es presentado como conocimiento objetivo y formal -la ambición del filósofo de las matemáticas-y lo que es realizado y sólo después formalizado por el matemático. El matemático comprende que desde el interior de lo que hace - Cantor y Dedekind de distinta manera pretendían dotar de fundamento cierto a la aritmética - requiere establecer con claridad enunciados generales y, con mucha frecuencia, no discute - ni se preocupa tanto, me atrevería a decir- por el carácter objetivo de sus afirmaciones. Insisto, otras preocupaciones le inquietan desde su quehacer particular, su actividad práctica específica, que además siempre avanza alumbrada de una intención explícita. En tal sentido, el rigor argumental está relacionado con la coherencia lógica de lo expuesto, no tanto con su carácter objetivo. Esta misma distinción es la que percibo en aquello que podemos denominar actividad política de insubordinación en marcha, distinta, aunque íntimamente relacionada, de la reflexión acerca de ella o - abusando de la analogía sugerida - con su formalización (Gutiérrez, 2005).
}

tanto la convivencia social y las "otras" posibilidades de su auto-regulación como las maneras de preservar y cuidar sus capacidades colectivas para asegurar la intervención autónoma y directa en los asuntos públicos, garantía, a fin de cuentas, de no ver detenido su propio avance. De ahí el lugar privilegiado que tiene por lo general la descripción detallada del acontecimiento de autounificación y lucha en mi trabajo. Sólo después de ello cabe un segundo orden lógico: el de la reflexión crítica de los significados explícitos y potenciales de las acciones y sucesos producidos por tales hombres y mujeres concretos.

En esa dirección, a la hora de seguir con cuidado los acontecimientos que se produjeron en esos años agitados y un poco después, en el esfuerzo por comprender la muchas veces contradictoria dinámica interna entre lo que efectivamente se emprendía como acción colectiva de lucha - manifestaciones, debates, bloqueos de caminos, levantamientos, cercos a las ciudades, etc.- y lo que se establecía como horizonte - como intención común a diputar y conseguir-, entendí la emancipación social ni principal ni únicamente como conjunto de objetivos explícitos y sistemáticos a concretar, sino como itinerario o trayectoria específica dificultosa, ambivalente, muchas veces contradictoria y particular, protagonizada por múltiples grupos, asociaciones, cuerpos y colectivos de hombres y mujeres concretos - fundamentalmente aquellos que no viven del trabajo ajeno- por eludir y confrontar la subordinación política y económica al orden instituido enmedio de diversos juegos móviles de tensiones y antagonismos. La reflexión sobre la emancipación consiste no tanto en dar cuenta objetiva de lo alcanzado y lo proyectado, sino en entender los caminos y obstáculos del potencial de transformación social anidado como desafío en la propia capacidad colectiva de incidir en el asunto público en un momento determinado. En cierto sentido, entiendo dicha dinámica de la lucha de emancipación como el contenido concreto y siempre abierto de una práctica política desplegada desde la autonomía política 
y sobre una cierta base mínima de autonomía material por quienes se proponen llevarla a cabo. ${ }^{15}$

Una vez delineadas en términos generales las consideraciones anteriores, me sumergiré en una discusión más específica de la práctica contemporánea de emancipación. Haciendo una reducción simplista que pido a los lectores me concedan para fines de la claridad del argumento, cabe la afirmación de que una parte de la discusión política contemporánea parece estar planteada así: política Estado-céntrica versus política autónoma. Es decir, se trata de una disyuntiva excluyente que establece la necesidad de una elección: o se realizan conjuntos de tareas y acciones para ocupar cargos públicos y "desde ahî", de "arriba hacia abajo", surge el compromiso para modificar algunas de las más opresivas relaciones sociales - conservando e intensificando otras-, o bien, esquemáticamente, se construye de forma múltiple y variopinta la capacidad colectiva y social por fuera del Estado, tanto para desarrollar y ampliar trechos de autonomía en la vida cotidiana como para impulsar luchas y poner límites a la devastación capitalista de la vida en general. Denomino provisionalmente a la primera finalidad "política autónoma auto-centrada" y a la segunda, "política autónoma expansiva". Estas dos formas no se presentan necesariamente en secuencia, en muchas ocasiones se mezclan y se confunden y en otras se distinguen con mayor claridad. Lo que es un hecho es que cualquier acción política autónoma expansiva se funda, necesariamente, en un trabajo asociado, colectivo y cooperativo que asegura la capacidad material de su despliegue, esto es, requiere de formas auto-centradas de política autónoma.

\footnotetext{
${ }^{15}$ La noción de emancipación presupone una relación anterior de sujeción, binaria o múltiple, que se rompe por decisión -y capacidad - de la parte anteriormente subordinada. Por lo general, la emancipación ha sido tratada sobre todo en sus aspectos "políticos", es decir, a partir de determinadas relaciones de poder instituidas, las cuales, desde la modernidad, se piensan en relación con el Estado y con el capital. Por mi parte, también relaciono la noción de emancipación a otra constelación de ideas: la autonomía, material y política, y la capacidad social práctica de establecer fines para sí misma.
}

Dicha disyunción epistémica excluyente entre política Estado-céntrica y política de y desde la autonomía casi nunca se presenta con tanta claridad en el terreno de la lucha concreta, sobre todo no aparece en todas las ocasiones como contradicción confrontada y en disputa, sino que se manifiesta con frecuencia como elección que distingue y separa a personas y grupos. Pensemos sobre esto con más detalle. Por lo general, después de grandes y extraordinarios momentos de lucha social y política que ponen en entredicho el orden político e institucional anterior, emerge la cuestión de si conviene "ocupar" cargos públicos para "consolidar" lo avanzado o si, por el contrario, en concordancia con la postura autonomista, conviene mantenerse fuera del Estado y reforzar las capacidades políticas alcanzadas desde la sociedad llana. Mi postura se inscribe claramente en la segunda perspectiva. El asunto es que, tal como he tratado de mostrar con los ejemplos presentados, en tales ocasiones se genera una gran confusión entre los militantes, pues la política "oficial" tiende hacia la seducción y cooptación de las perspectivas y asociaciones autónomas, o bien hacia su perversión, devaluación, desplazamiento y anulación. Además, dentro del propio cuerpo social autónomo movilizado cristaliza la discusión acerca de lo que conviene hacer en relación con el poder estatal.

Tengo la impresión de que la claridad de tal disyunción de fondo no resuelve demasiados problemas sino que nos abre otra amplia gama de interrogantes. La primera es qué se hace, como precisa la mencionada "formulación de Cochabamba", desde una política de la autonomía respecto del poder establecido. Qué se hace, una vez más, no sólo en términos de teoría o crítica, sino en torno a la manera específica en que tal poder se ejerce. Qué se hace en relación con los pasos particulares que dan tanto gobernantes y funcionarios estatales como administradores y gestores de la acumulación de capital. Cómo reconocemos lo que nosotros mismos hemos hecho - desde nuestra propia política-, que a veces incluye que ellos hayan tenido que variar o desacelerar, por poco que sea, sus planes y proyectos políticos. Se 
trata de reconocerlo para profundizarlo, no para intentar sustituirlo. Éste es un asunto de la mayor relevancia pues, si desde la política de la autonomía se abandona la voluntad y disposición de establecer "qué ha de hacerse" con los asuntos que a algunos o a todos incumben, de modo que tendencialmente se busque "mandatar" a quienes ocupen cargos públicos, si se abandona esa capacidad y esa posición simbólica - que se recupera por lo general en momentos de confrontación, de despliegue del antagonismo social-, entonces se permite que el entramado normativo e institucional y los funcionarios que lo habitan vuelvan a ocuparlo, desplazando a la "gente sencilla y trabajadora" hacia su tradicional lugar de obediencia y soporte de decisiones ajenas. ${ }^{16}$

En términos teóricos, tengo dudas acerca de qué significa admitir que la relación entre la política Estado-céntrica y la política desde la autonomía sea de disyunción. ¿Significa esto que necesariamente la relación entre ambas se da en términos de confrontación? ¿O significa que afirmamos una diferencia incompatible de perspectiva? Por lo pronto, elijo la segunda connotación, es decir, que estas dos formas de política son distintas desde su fundamento, que se proponen objetivos que corren por cauces diferentes y que, por tanto, deberíamos abordar la cuestión de esa manera. La disyunción establece que se trata de dos perspectivas políticas distintas e incomparables. Inconmensurables podría decir el matemático, proceden y se ocupan de cuestiones diferentes, en ocasiones se confrontan frontalmente, aunque en todo caso esto no ocurre siempre y sobre todo no ocurre necesariamente.

Analicemos, para intentar contribuir a aclarar este aspecto, en qué se distinguen ambas posturas. Una de las primeras claves es que cada posición enuncia lo que propone de forma incompatible,

${ }^{16}$ Abordé esta cuestión en Los ritmos del Pachakuti (Gutiérrez, 2009) pensando en las distintas maneras en que se quebró el orden de mando en Bolivia entre 2000 y 2005. Cuando ese orden colapsa paulatinamente, con base en la capacidad de despliegue del antagonismo desde abajo, se produce una inversión tendencial entre quienes mandan y quienes obedecen. La propia noción de Pachakuti alude, aunque no únicamente, a tal colapso e inversión. pese a que quizá utilicen palabras similares. La política Estado-céntrica enuncia lo que hará para y sobre el conjunto de la sociedad: qué tipo de políticas implementará, qué programas de redistribución desarrollará, etc. Al hablar de esa manera se sitúa simbólicamente en el lugar de la representación aparente de la totalidad social y pretende que habla "para todos". Es el sitio de enunciación universal afirmativo desde donde el poder-imposición predominantemente masculino ha hablado siempre. Y desde ahí no puede decirse más que lo que se ha dicho, aunque existan algunos matices.

En contraste, la política de la autonomía es, siempre, concreta y particular, si bien puede ser expansiva o auto-centrada, según lo requieran quienes la echen a andar y en qué momentos lo hagan. La política de la autonomía es concreta y particular porque, en principio, habla en primera persona: "nosotros nos proponemos hacer tal o cual cosa... y vamos a hacerlo de esta manera", explica cuando se trata de un momento auto-centrado de la política autónoma; "nosotros consideramos que tal o cual cosa que incumbe a todos debe realizarse de esta $u$ otra manera...", se establece en momentos expansivos. La segunda distinción que conviene tener a la vista es que la política Estado-céntrica razona desde la perspectiva de la estabilización del sistema de fuerzas y tensiones antagónicas cuyo gobierno ya ha ocupado o busca ocupar. Su tarea fundamental es estabilizar y conservar, así ofrezca y prometa que una vez alcanzado algún tipo de equilibrio alentará o introducirá transformaciones en el orden de la acumulación de capital y en el ejercicio del mando político. Una política de emancipación desde la autonomía, por su parte, sobre todo en sus momentos expansivos, suele orientarse por la búsqueda de la desestabilización parcial, de la apertura particular y concreta de aquellas normas e instituciones que impiden su despliegue.

A partir de las consideraciones sobre aquello que distingue a una política de emancipación desde la autonomía de la política Estado-céntrica podemos discutir algunas nociones que han adquirido el formato 


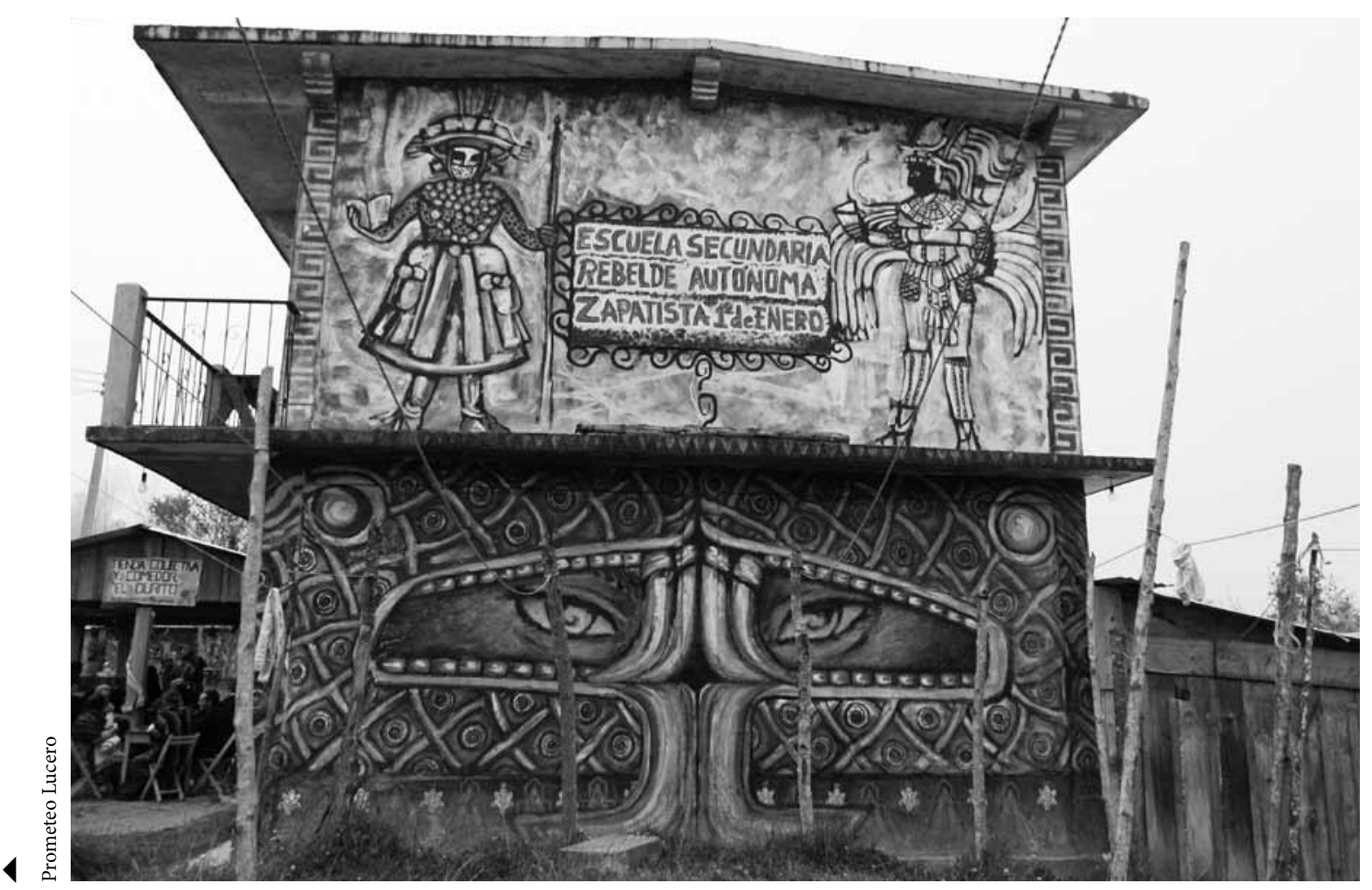

Oventik, Chiapas, enero de 2009.

de prejuicio confuso en los tiempos recientes. La primera es que desde la política de la autonomía nunca puede establecerse contacto con lo instituido, ni con el Estado ni con el capital. La validez parcial de esta idea brota desde la más legítima y fundada desconfianza en todo lo que huela a disciplina y a sujeción, $y$ puede llegar a constituirse como un prejuicio nocivo si se determina como criterio exterior y universal, que limita de antemano cualquier despliegue particular de la política autónoma. Por más radical que sea la afirmación del rechazo a lo estatal/capitalista, formulada de la manera anterior se convierte en un límite para el despliegue de la política autónoma. Insisto, en ningún sentido estoy diciendo que la política desde la autonomía deba "enredarse" sistemáticamente con las formas y modos del Estado y del capital. Sencillamente sostengo que la política autónoma no puede admitir límites exteriores y anteriores a su propio despliegue y decisión. Regreso sobre el asunto de que los enunciados universales y prescriptivos hablan, siempre, desde el locus del poder-imposición (Colectivo Situaciones, 2010).

El segundo prejuicio no tiene una figura expresiva tan explícita, pero empaña los razonamientos de quienes se esfuerzan por desarrollar una política autónoma. Me refiero al asunto del frecuente olvido -ingenuo o interesado- de las condiciones materiales de la autonomía, que aleja la discusión política de su carácter concreto para reinstalarla en los terrenos nebulosos de la coherencia abstracta. Así, en una política desde la autonomía no se trata de prescribir lo que "debe" hacerse en general, sino de reflexionar en profundidad sobre las dificultades eminentemente prácticas del camino a recorrer para construir lo que se proyecta en común y de encontrar cada vez, procurando no perderse, maneras de sortear obstáculos. 


\section{IV}

Para finalizar, formulo algunas consideraciones sobre otro asunto pertinente. Cabe afirmar, con base en la argumentación anterior, que hasta cierto punto la política de emancipación desde la autonomía obliga a que se desplace en el orden del pensamiento y el debate otra vieja disyuntiva de principios del siglo $\mathrm{xx}$, que continúa opacando la comprensión del evento político. Me refiero a la disyuntiva entre reformismo y revolución. Éstas eran distinciones acuñadas para designar variantes de la política Estado-céntrica. ¿Qué ocurre, sin embargo, si rechazamos la disyunción entre reforma y revolución, por imposible y contrapuesta a las luchas por la emancipación, en tanto no pueden transformarse las relaciones sociales desde arriba hacia abajo, ni paulatina ni abruptamente? ¿Qué ocurre si, al mismo tiempo, nos planteamos de manera sistemática las cuestiones de la disolución y el trastocamiento de las relaciones de poder del capital y del Estado que están ahí, exigiendo respuestas a una política desde la autonomía?

Si damos ese paso, entonces somos nosotros mismos quienes realizamos un desplazamiento en el orden simbólico dominante y desde ahí, desde ese frágil y dificultoso sitio propio, podemos eludir, o al menos tratar de esquivar, en mejores condiciones, las dificultades que el Estado y el capital imponen a nuestra práctica política y a su expansión. Desde ahí podemos intervenir en los asuntos públicos y "hacer política”, exponer nuestros puntos de vista y dialogar acerca de nuestras necesidades e ideas, sin acudir jamás al Estado en términos de demandantes -que es donde siempre busca colocarnos-, eso sí, habilitando un terreno para establecer las veces que así lo requiramos, con claridad y fuerza, lo que éste debe o no debe hacer. Podemos hablar y hacer política desde este lugar de una manera "reformista" o "revolucionaria", es decir, en ocasiones podemos adelantar propuestas prácticas y definir plazos para llevarlas a cabo de manera progresiva y, si se considera necesario, simultáneamente confrontando o no, inmediata y directamente, al poder público. Por lo general, no tomamos solos muchas de esas decisiones, sino que una parte de ellas se nos imponen: ellos nos atacan y nosotros nos defendemos.

Es en este sentido que entiendo la política de emancipación desde la autonomía: como trayectoria, como producción sistemática de posibilidad abierta, como esfuerzo reiterado de no caer en las trampas de la totalidad. La dificultad para abordar estas cuestiones está no sólo en su fluidez y, por lo mismo, en la necesaria ductilidad y apertura del pensamiento que requerimos para situarnos ante ellas, sino en que son problemas esencialmente prácticos que se presentan casi siempre al calor de los acontecimientos de despliegue del antagonismo social. Es decir, ocurren vertiginosamente al interior del primer orden de intelección del problema, enmedio de la lucha misma. En tal dirección, los inciertos elementos que podamos esbozar sobre estos temas, instalados, como es el caso actual, en el segundo orden, en el de la reflexión sobre el acontecimiento político y la lucha social, siempre serán provisionales y tentativos. Desde este terreno no podemos realizar más que efímeros esfuerzos sintéticos parciales.

Así, entendiendo la auto-emancipación como el contenido particular y concreto de una política desde la autonomía, queda pendiente y abierto el asunto de la disolución y trastocamiento del poder y del capital, desanudando y rasgando los nudos que le dan fuerza, alterando sus reglas, inhibiendo su enloquecida acumulación. Mirando desde lo que las sociedades en movimiento efectivamente hicieron durante las grandes luchas que inauguraron el siglo presente, la mencionada "cuestión del poder" adquiere otro sentido: se trata de pensar en y ocuparse de cuidar y expandir, de múltiples maneras, la capacidad autónoma para intervenir en los asuntos públicos alcanzada en los tiempos turbulentos, de empecinarse en no ceder el lugar de enunciación propio, construido dificultosamente, que erosiona y escapa de los conceptos y del canon argumental de la dominación y explotación capitalista y neoliberal de la vida y sus 
posibilidades múltiples de creación y producción. Se trata, entonces, tal como dice la sabiduría femenina aymara, de que "entendamos" el tamaño y la fuerza de nuestra propia capacidad y de que no la rindamos ante nadie - así se presente ante nosotros como "nuestro marido"- La cara oculta del poder y del capital es la sujeción de la capacidad de pensar y hacer de todos los que no son él mismo. La recuperación y recreación cotidiana de esas capacidades para nosotros mismos es la medida del debilitamiento y disolución del otro poder. Ahí hay un mar de tareas $\mathrm{y}$ cuestiones pendientes.

Además, y a manera de resumen, la perseverancia en una política de emancipación orientada por la conservación y expansión de las capacidades sociales autónomas ya alcanzadas o conservadas, íntimas y colectivas, para el despliegue de la vida más allá y contra el capital y tendencialmente hacia la regulación autónoma del asunto común, necesariamente ha de pensarse desde el punto de vista de lo particular y de la inestabilidad del orden existente. La peor trampa para la política autónoma de la emancipación es confundirse y suponer que quienes hablan desde el gobierno o desde el Estado tienen razón cuando exigen a la sociedad llana - $\mathrm{o}$ a quienes luchan desde ese lugar- que asuman el punto de vista de la totalidad social y de la estabilización de un orden pretendidamente "nuevo". Mientras la sociedad esté desgarrada por brutales antagonismos como lo está ahora, la política de la emancipación habrá de trastocar el orden que se le impone desde los múltiples particulares que genera y habita. La emancipación en tal sentido es camino y trayecto, es esfuerzo por esclarecer los itinerarios y por ampliar y des-sujetar los flujos de la energía social que, a fin de cuentas, son el fundamento de cualquier creación de novedad.

\section{Bibliografía}

Colectivo Situaciones, 2010, Conversaciones en el impasse. Dilemas políticos del presente, Universidad Internacional de Andalucía, Tinta Limón, Buenos Aires.

Gutiérrez, Raquel, 2005, "En torno a la naturaleza de las proposiciones de la aritmética y la noción de número: Mill, Frege, Cantor y Dedekind", tesis de maestría en filosofía, Facultad de Filosofía y Letras-Universidad Nacional Autónoma de México, México.

- 2006, $; A$ desordenar! Por una historia abierta de la lucha social, Centro de Estudios Andinos y Mesoamericanos, Juan Pablos, México.

- 2009, Los ritmos del Pachakuti. Movilización y levantamiento en Bolivia 2000-2005, Instituto de Ciencias Sociales y Humanidades-Benemérita Universidad Autónoma de Puebla, Bajo Tierra Ediciones, México.

Gutiérrez, Raquel y Dunia Mokrani, 2009, "Bolivia a un año del gobierno de Evo Morales: elementos para un balance", en Nayar López Castellanos y Lucio Oliver (coords.), América Latina y el Caribe: una región en conflicto, Facultad de Ciencias Políticas y Sociales-Universidad Nacional Autónoma de México, Plaza y Valdés, México.

Holloway, John, 2001, Cambiar el mundo sin tomar el poder, Herramienta, Benemérita Universidad Autónoma de Puebla, Buenos Aires.

Mamani, Pablo, Lucila Choque y Abraham Delgado, 2010, Memorias rebeldes. Reconstitución de Tupaj Katari y Bartolina Sisa. ¡Somos millones!, Textos Rebeldes, Pez en el Árbol, Willka, La Paz.

Tapia, Luis Fernando, Dunia Mokrani y Patricia Chávez, 2007, "Democracia y cambio político en Bolivia", documento de trabajo, Programa de Investigación Estratégica en Bolivia, La Paz. 